\title{
CONF- $960493--5$ \\ Thermal Study of Silicon Optical Microbenches With on Board Heaters for Soldering
}

M.D. Pocha

O.T. Strand

J.A. Kerns

This paper was prepared for submittal to the

Optical Society of America

Topical Meeting on Integrated Photonics Research

Boston, MA

April 29-May 3, 1996

January 1996

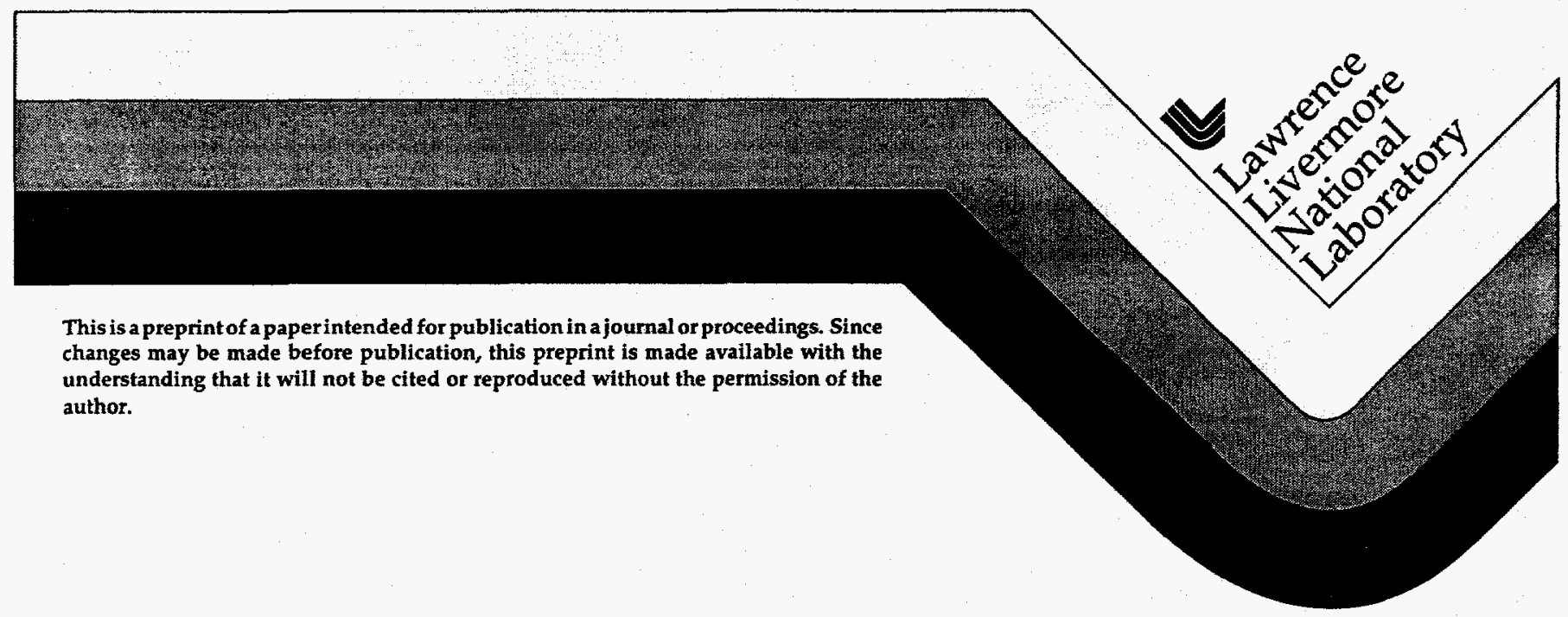

DISTAIBITION OF THIS DOCUMENT IS UNLMTIED 


\section{DISCLAIMER}

This document was prepared as an account of work sponsored by an agency of the United States Government. Neither the United States Government nor the University of California nor any of their employees, makes any warranty, express or implied, or assumes any legal liability or responsibility for the accuracy, completeness, or usefulness of any information, apparatus, product, or process disclosed, or represents that its use would not infringe privately owned rights. Reference herein to any specific commercial product, process, or service by trade name, trademark, manufacturer, or otherwise, does not necessarily constitute or imply its endorsement, recommendation, or favoring by the United States Government or the University of California. The views and opinions of authors expressed herein do not necessarily state or reflect those of the United States Government or the University of California, and shall not be used for advertising or product endorsement purposes. 


\section{DISCLAIMER}

Portions of this document may be illegible in electronic image products. Images are produced from the best available original document. 


\title{
Thermal Study of Silicon Optical Microbenches with on Board Heaters for Soldering
}

\author{
Michael D. Pocha \\ Lawrence Livermore National \\ Laboratory \\ P.O. Box 808, L-222 \\ Livermore, CA 94550 \\ (510) $422-8664$ \\ FAX (510) 422-2783
}

\author{
Oliver T. Strand \\ Lawrence Livermore National \\ Laboratory \\ P.O. Box 808, L-174 \\ Livermore, CA 94550 \\ (510) 423-2062 \\ FAX (510) 422-1066
}

\author{
John A. Kerns \\ Lawrence Livermore National \\ Laboratory \\ P.O. Box 808, L-287 \\ Livermore, CA 94550 \\ (510) 422-9586 \\ FAX (510) 422-6747
}

\begin{abstract}
The combination of selected experimental measurements with finite element simulation provide a thorough understanding of thermal behavior of silicon microbenches with on-board heaters.
\end{abstract}




\title{
Thermal Study of Silicon Optical Microbenches with on Board Heaters for Soldering
}

\author{
Michael D. Pocha, Oliver T. Strand and John A. Kerns \\ Lawrence Livermore National Laboratory \\ P.O. Box 808, L-222, Livermore, CA 94550 \\ (510) 422-8664, FAX (510) 422-2783
}

\section{Introduction}

High-precision packaging of optoelectronic $(\mathrm{OE})$ components is a laborintensive and expensive process. We believe that the pigtailing process must be automated to realize a significant reduction in the cost of $O E$ packages. We are addressing issues of automating the fiber pigtailing process on silicon waferboards or microbenches. While we are initially concentrating on laser diodes, the same techniques are applicable to other $\mathrm{OE}$ devices. This paper focuses on reflowing solders for the attachment of OE components on silicon microbenches. Nearly ten years ago, miniature metallic heaters to reflow solder for the attachment of $\mathrm{OE}$ components on ceramic waferboards were developed [1]. We have recently developed miniature polysilicon heaters which are compatible with silicon microbenches [2]. These miniature heaters avoid the problem of raising the entire microbench to the solder melting point to attach components. Most importantly, these miniature heaters are completely compatible with automating the attachment process.

Designing silicon microbenches with on-board heaters requires some care. The thermal properties of the microbench itself along with all coatings on the surface and any heatsinking materials must be understood. The heaters must òperate in a current and voltage regime compatible with the overall characteristics of the $\mathrm{OE}$ package. Inadvertently reflowing solder in unanticipated locations may occur unless the thermal behavior of the microbench is thoroughly known. This paper, then, describes an experimental and theoretical study we have performed on these silicon microbenches which gives us a complete picture of their thermal behavior. We have two goals. First, we want to understand the temperature distribution on our existing microbench designs. Secondly we want to develop a computational tool to model the thermal behavior and help us to design future microbenches. The next section describes the geometry of our existing silicon microbenches and the following section describes the theoretical thermal model we are using and simulations to extend our experimental results. The final sections present the results of the experiments and their comparison with the model.

Structure and construction of
microbenches

Construction of our silicon microbenches begins with single-crystal silicon wafers as a substrate. We use a standard silicon wafer thickness of $400-500 \mu \mathrm{m}$. Wells and pedestals are etched into the silicon as needed to approximately align, in the vertical direction, the optical axis of the various components to be mounted. Next a thick layer of silicon dioxide $\left(\mathrm{SiO}_{2}\right)$ is deposited and etched to provide some degree of thermal isolation of other areas from the heaters. The thermal resistance of $\mathrm{SiO}_{2}$ is approximately two orders of magnitude greater than that of silicon so a $5 \mu \mathrm{m}$ thick layer of $\mathrm{SiO}_{2}$ is thermally equivalent to a $500 \mu \mathrm{m}$ thick layer of silicon. With plasmaassisted Chemical Vapor Deposition, it is possible to deposit layers of $\mathrm{SiO}_{2}$ up to 20$30 \mu \mathrm{m}$ thick. We generally use a thickness of 5 $10 \mu \mathrm{m}$ depending on the needs of each application.

The heaters are made by depositing, doping, and patterning an approximately $1 \mu \mathrm{m}$ thick layer of polycrystalline silicon (polysilicon). The doping is adjusted to result in a resistance of the heaters of approximately 10-50 $\Omega$. Resistors in this range give the greatest control at the temperatures of interest with standard laboratory power supplies. A final metal layer of a standard $\mathrm{Cr} / \mathrm{Au}$ or $\mathrm{Ti} / \mathrm{Au}$ metallization completes the interconnect 
structure to provide electrical connections to the heaters and a layer of metal that is strongly adherent to both silicon and $\mathrm{SiO}_{2}$ and to which most standard solders will adhere.

Figure 1 shows the top view of a typical microbench. The overall dimensions are about $6 \mathrm{~mm} \times 13 \mathrm{~mm}$. The spacing between the mounting location of the laser diode and the nearest heater is approximately $1 \mathrm{~mm}$ and the second heater is about $5 \mathrm{~mm}$ away from the first. By judicious choice of solders and power to the heaters we can solder the laser diode, a thermistor, and metal coated fiber to the top as well as a thermoelectric cooler to the bottom of the microbench with just these two heaters. Using this technique we have been able to achieve sub-micron tolerance of conically tapered fiber actively aligned and attached with coupling efficiencies as high as $65 \%$.

\section{Thermal Modeling and Simulation}

A mechanical finite-element modeling code called COSMOS/M[3] was used to develop a predictive model of the temperature response of the silicon microbench to various heating pulses. Full three dimensional simulation is needed to account for all the heat flow in the structure, however we found that we could simplify the model by approximating the three dimensional physical structure as a two dimensional axisymmetric problem. This was justified because the microbench is thin compared to its overall width and length dimensions. Heat penetrates quickly through the thin wafer and then spreads radially outward. Our strategy here was to compare the model to measured results at two extremes of heatsinking. First, heat sinking only around the edge of the microbench simulates minimum cooling where just natural convection of air provides the cooling. Second, heat sinking under the entire microbench provides much larger thermal losses and simulates the attachment of the microbench to a real package. For comparison with our experiments, we modeled different types of heatsink materials including glass, brass, and a thermal-electric cooler. We did not permanently solder the microbenches to the heatsinks, so that the benches and the heatsinks could be reused. The microbenches were attached to the heat sinks by either a thin layer of silicone grease or thermal epoxy.

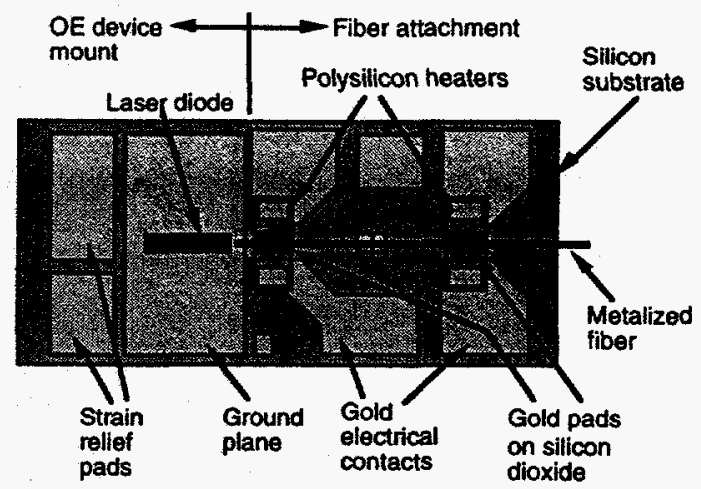

Figure 1. Silicon microbench with built-in polysilicon heaters for manufacturable solder reflow.

Because the edge boundaries of the microbench are relatively far from the heat source we can use a circular heat source in the model to approximate the actual rectangular heat source. This gives accurate central temperatures, relative edge temperatures at the source boundary, and accurate temperatures far from the source. Local temperature prediction at the heater determines the melt or remelt of the solder, while temperatures far from the

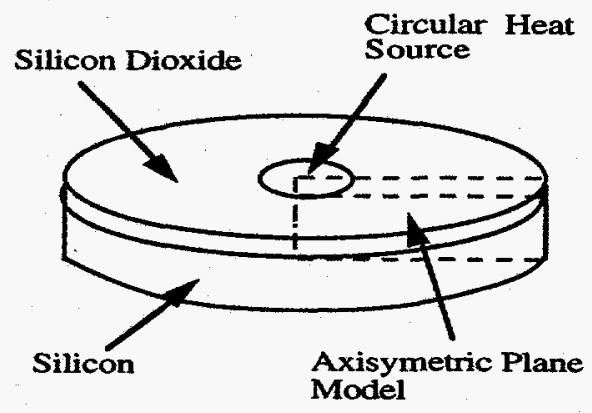

Figure 2. Thermal model of the silicon microbench showing the cylindrical approximation to the physical model and the planar section used in the simulation. Heat is removed by natural convection from the top surface and by a heat sink located under the microbench.

heater determine the fate of the other solder junctions on the microbench.

The thermal model developed for the microbench assumes axial symmetry and is a plane section cut radially from a cylindrical piece of silicon. A sketch of the axisymmetric 
model is shown in Figure 2. The height of the rectangular plane is equal to the thickness of the silicon $(450 \mu \mathrm{m})$ plus a $25 \mu \mathrm{m}$ height that approximates the $5 \mu \mathrm{m}$ insulation layer of silicon dioxide. The insulation layer was purposely made thicker so that fewer nodes would be required in the simulation. The material properties of the insulation were adjusted to match the thermal properties of the $5 \mu \mathrm{m}$ silicon dioxide layer. Natural convection heat transfer removes some heat from the surface of the microbench but the majority of the heat is removed through contact resistance with heat sinks located near the bottom edges of the microbench or under the microbench. The contact resistance between the microbench and a heat sink is a large thermal barrier to heat flow. This resistance is difficult to predict accurately because of uncertainties in the gap height between contacting parts. For this study, the contact resistance was the only variable in the model used to match the data.

We are running COSMOS/M on a Macintosh II with a $16 \mathrm{MHz} 68030$ processor and a math co-processor. On this somewhat primitive platform we can perform steady state simulations in less than 10 minutes and transient simulations in less than $\mathbf{3 0}$ minutes.

\section{Experimental Setup}

The experimental data was gathered using a flexible IEEE-488 bus controlled measurement system. Measurements were taken of temperature vs. time with a variety of applied electrical power pulses and at several locations on the microbench. For transient measurements shorter than one second we found that it was necessary to attach miniature chip thermistors to the microbench. The thermal response time of even the smallest thermocouple we had available was too long (several seconds). Thermistors, however, are restricted to a temperature range below $150^{\circ} \mathrm{C}$. So, for short pulses our experimental data is restricted to that range. We can extrapolate the experimental data to higher temperatures with the computer simulations. Three chip thermistors were mounted on each microbench tested: one on top of the central heater, the next approximately $2 \mathrm{~mm}$ away on the diode mounting pad, and the third on one of the strain relief pads about $5 \mathrm{~mm}$ from the heater.

\section{Experimental Results}

Due to limitation of space, only a few sample results are described here; additional results

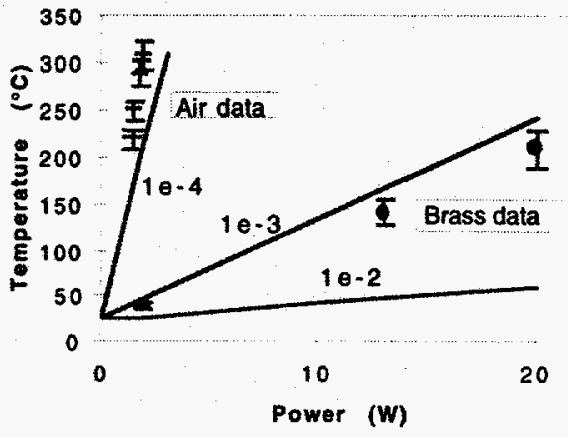

Figure 3. Simulated and experimental plot of steady state temperature with various values of cooling coefficients to represent heatsinking.

will be presented at the conference. Figure 3 shows a plot of steady state temperature vs. electrical power applied to the heater for several different cooling coefficients in units of $\mathrm{W} / \mathrm{mm}^{2}-{ }^{\circ} \mathrm{C}$. The experimental data allows determination of the effective cooling coefficients. From such plots we can calibrate the model for a particular cooling mechanism. Using this model, we can make this kind of plot for any location on the microbench.

Figure 4 shows the decrease in temperature with distance away from a heater

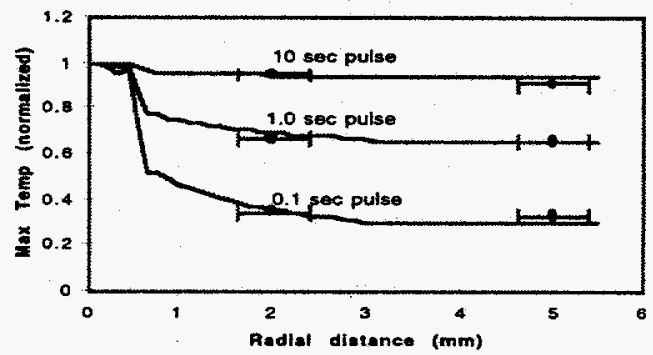

Figure 4. Typical plot of normalized temperature vs. radial position for various lengths of electrical pulses applied to the heater

for different electrical pulse lengths. This information allows one to determine how far 
apart to design the attachment points of components which must be soldered sequentially in time. One wants to maintain the first component at a temperature lower than the melting point of the solder while attaching subsequent components. As one would expect, a short pulse results in a larger decrease in temperature with distance because the heat is initially concentrated only at the heater.

\section{Conclusion}

An experimental and theoretical study has been conducted of the thermal behavior of silicon microbenches with on-board heaters for solder reflow. The combination of selected experimental measurements with finite element simulation of the thermal behavior of the structure allows us to gain a good understanding of temperature distributions on the microbench under various conditions of power applied to heaters and for different heatsinks. The result is an accurate predictive model of the silicon microbench.

\section{Acknowledgments}

The authors would like to thank their colleagues Leslie Jones, Kirk Kleint, and Ronald Tilley for help in fabricating microbenches and taking experimental data. This work was performed under the auspices of the U.S. Department of Energy by Lawrence Livermore National Laboratory under contract No. W-7405-Eng-48.

\section{References}

1. S. Enochs, "A packaging technique to achieve stable single-mode fiber to laser alignment," SPIE Integration and Packaging of Optoelectronic Devices, Vol. 703, p 42 (1986).

2. M. Lowry, et. al., "Low-Cost Packaging of High-Performance Optoelectronic Components," IPR-95 Technical Digest, Vol. 7, pp.77-79, Feb. 23-25, 1995

3. Available from Structural Research Analysis Corp., 1661 Lincoln Blvd., Suite 200, Santa Monica, CA 90404, Phone: (310) 452-2158 